\title{
$q$-Lidstone polynomials and existence results for $q$-boundary value problems
}

\section{Zeinab Mansour ${ }^{1,2}$ and Maryam Al-Towailb ${ }^{3 *}$}

\section{"Correspondence:}

mtowaileb@ksu.edu.sa

${ }^{3}$ Department of Natural and Engineering Science, Faculty of Applied Studies and Community Service, King Saud University, Riyadh, Kingdom of Saudi Arabia Full list of author information is available at the end of the article

\section{Abstract}

In this paper, we study some properties of $q$-Lidstone polynomials by using Green's function of certain $q$-differential systems. The $q$-Fourier series expansions of these polynomials are given. As an application, we prove the existence of solutions for the linear q-difference equations

$$
(-1)^{n} D_{q^{-1}}^{2 n} y(x)=\phi\left(x, y(x), D_{q^{-1}} y(x), D_{q^{-1}}^{2} y(x), \ldots, D_{q^{-1}}^{k} y(x)\right),
$$

subject to the boundary conditions

$$
D_{q^{-1}}^{2 j} y(0)=\beta_{j,} \quad D_{q^{-1}}^{2 j} y(1)=\gamma_{j} \quad\left(\beta_{j}, \gamma_{j} \in \mathbb{C}, j=0,1, \ldots, n-1\right),
$$

where $n \in \mathbb{N}$ and $0 \leq k \leq 2 n-1$. These results are a $q$-analogue of work by Agarwal and Wong of 1989.

MSC: $05 \mathrm{~A} 30 ; 11 \mathrm{~B} 68 ; 39 \mathrm{~A} 05 ; 39 \mathrm{~A} 13 ; 30 \mathrm{E} 25 ; 42 \mathrm{~A} 16$

Keywords: $q$-difference equations; Green's function; $q$-Lidstone polynomials; q-Fourier expansions

\section{Introduction}

In the classical Lidstone expansion theorem [1], an entire function $f(x)$ may be expanded with respect to the points 0 and 1 in the form

$$
f(x)=\sum_{n=0}^{\infty}\left(f^{(2 n)}(1) A_{n}(x)-f^{(2 n)}(0) A_{n}(x-1)\right),
$$

where $A_{n}$ is a polynomial of degree $2 n+1$ that satisfies

(i) $A_{0}(x)=x$

(ii) $A_{n}(0)=A_{n}(1)=0$ for $n \in \mathbb{N}$,

(iii) $A_{n}^{\prime \prime}(x)=A_{n-1}(x)$.

The polynomial $A_{n}$ is called Lidstone polynomial.

Ismail and Mansour [2] introduced a $q$-analogue of Lidstone's theorem where the two points are 0 and 1 . They expanded the function in $q$-analogues of Lidstone polynomials which are in fact $q$-Bernoulli polynomials as in the classical case (see Section 2).

(c) The Author(s) 2017. This article is distributed under the terms of the Creative Commons Attribution 4.0 International License (http://creativecommons.org/licenses/by/4.0/), which permits unrestricted use, distribution, and reproduction in any medium, provided you give appropriate credit to the original author(s) and the source, provide a link to the Creative Commons license, and indicate if changes were made. 
It is the object of this paper to give a $q$-analogue of the results of [3] using the terminology and results given in [2].

This article is organized as follows. In the next section, we state the $q$-definitions and present some preliminaries of $q$-calculus which will play an important role in our main results. In Section 3, we define the Green's functions of certain $q$-differential systems which are related to $q$-Lidstone polynomials, and Section 4 gives $q$-Fourier expansions of these functions and for $q$-Lidstone polynomials. Some interesting results and relationships are obtained. In Section 5, we are interested in the existence of solutions to the following boundary value problem:

$$
(-1)^{n} D_{q^{-1}}^{2 n} y(x)=\phi\left(x, y(x), D_{q^{-1}} y(x), D_{q^{-1}}^{2} y(x), \ldots, D_{q^{-1}}^{k} y(x)\right)
$$

$n \in \mathbb{N}$ and $0 \leq k \leq 2 n-1$, subject to the boundary conditions

$$
D_{q^{-1}}^{2 j} y(0)=\beta_{j}, D_{q^{-1}}^{2 j} y(1)=\gamma_{j} \quad\left(\beta_{j}, \gamma_{j} \in \mathbb{C}, j=0,1, \ldots, n-1\right)
$$

with some conditions imposed on $y$.

\section{Preliminaries}

In this paper, we assume that $q$ is a positive number less than one with

$$
[x]=\frac{1-q^{x}}{1-q} .
$$

For $t>0$, the sets $A_{q, t}, A_{q, t}^{*}$ are defined by

$$
A_{q, t}:=\left\{t q^{n}: n \in \mathbb{N}_{0}\right\}, \quad A_{q, t}^{*}:=A_{q, t} \cup\{0\},
$$

where $\mathbb{N}_{0}:=\{0,1,2, \ldots\}$. Notice, if $t=1$, we simply use $A_{q}$ and $A_{q}^{*}$ to denote $A_{q, 1}$ and $A_{q, 1}^{*}$, respectively.

In the following, we state some of the needed $q$-notations and results (see [4] and [5]).

The $q$-shifted fractional is defined by

$$
(a ; q)_{\infty}=\prod_{j=0}^{\infty}\left(1-a q^{j}\right) \quad \text { and } \quad(a ; q)_{n}:=\frac{(a ; q)_{\infty}}{\left(a q^{n} ; q\right)_{\infty}} \quad \text { for } n \in \mathbb{Z}, a \in \mathbb{C}
$$

The $q$-gamma function is defined by

$$
\Gamma_{q}(z)=\frac{(q ; q)_{\infty}}{\left(q^{z} ; q\right)_{\infty}}(1-q)^{1-z} \quad \text { for } z \in \mathbb{C} \backslash\left\{-n: n \in \mathbb{N}_{0}\right\}
$$

Let $f$ be a function defined on a $q$-geometric set $A$, i.e., $q x \in A$ for all $x \in A$. The $q$ difference operator is defined by

$$
D_{q} f(x):=\frac{f(x)-f(q x)}{(1-q) x} \quad \text { if } x \in A-\{0\}
$$


The $q$-integration by parts rule (see [4]) is

$$
\int_{0}^{a} f(q t) D_{q} g(t) d_{q} t=(f g)(a)-\lim _{n \rightarrow \infty}(f g)\left(a q^{n}\right)-\int_{0}^{a} D_{q} f(t) g(t) d_{q} t .
$$

If $X$ is the set $A_{q, t}$ or $A_{q, t}^{*}$, then for $n>1, C_{q}^{n}(X)$ is the space of all continuous functions with continuous $q$-derivatives up to order $n-1$ on $X$. The space $C_{q}^{n}(X)$ associated with the norm function

$$
\|f\|:=\sum_{k=0}^{n-1} \max _{x \in X}\left|D_{q}^{k} f(t)\right| \quad\left(f \in C_{q}^{n}(X)\right)
$$

is a Banach space (see [4]).

Ismail and Mansour [2] defined a $q$-analogue of the Bernoulli polynomials $B_{n}(z ; q)$, $z \in \mathbb{C}$ by the generating function

$$
\frac{t E_{q}(z t)}{E_{q}(t / 2) e_{q}(t / 2)-1}=\sum_{n=0}^{\infty} B_{n}(z ; q) \frac{t^{n}}{[n] !}
$$

where the functions $E_{q}(z)$ and $e_{q}(z)$ have the series representation

$$
e_{q}(z)=\sum_{k=0}^{\infty} \frac{z^{k}}{\Gamma_{q}(k+1)} ; \quad|z|<1 \quad \text { and } \quad E_{q}(z)=\sum_{k=0}^{\infty} \frac{q^{k(k-1) / 2} z^{k}}{\Gamma_{q}(k+1)} ; \quad z \in \mathbb{C} .
$$

The $q$-Bernoulli numbers are defined by

$$
\beta_{n}:=B_{n}(0 ; q)
$$

Hence, in terms of the generating function,

$$
\frac{t}{E_{q}(t / 2) e_{q}(t / 2)-1}=\sum_{n=0}^{\infty} \beta_{n} \frac{t^{n}}{[n] !}
$$

Also, they defined two $q$-analogues of the Euler polynomials through the generating functions

$$
\begin{aligned}
& \frac{2 E_{q}(x t)}{E_{q}(t / 2) e_{q}(t / 2)+1}=\sum_{n=0}^{\infty} E_{n}(x ; q) \frac{t^{n}}{[n] !}, \\
& \frac{2 e_{q}(x t)}{E_{q}(t / 2) e_{q}(t / 2)+1}=\sum_{n=0}^{\infty} e_{n}(x ; q) \frac{t^{n}}{[n] !} .
\end{aligned}
$$

Notice, $E_{0}(x ; q)=e_{0}(x ; q)=1$, and $\tilde{E}_{n}:=E_{n}(0 ; q)=e_{n}(0 ; q)$ for all $n \in \mathbb{N}_{0}$.

Proposition 2.1 For $n \in \mathbb{N}$, the q-Bernoulli and q-Euler polynomials satisfy the following q-difference equations:

$$
\begin{aligned}
& D_{q^{-1}} B_{n}(x ; q)=[n] B_{n-1}(x ; q) ; \\
& D_{q^{-1}} E_{n}(x ; q)=[n] E_{n-1}(x ; q) \text { and } D_{q} e_{n}(x ; q)=[n] e_{n-1}(x ; q) .
\end{aligned}
$$


Proposition 2.2 The $q$-Euler polynomials $E_{n}(x ; q)$ and $e_{n}(x ; q)$ are given by

$$
E_{0}(x ; q)=e_{0}(x ; q)=1
$$

and for $n \in \mathbb{N}$,

$$
E_{n}(x ; q)=\sum_{k=0}^{n}\left[\begin{array}{l}
n \\
k
\end{array}\right]_{q} q^{\frac{k(k-1)}{2}} \tilde{E}_{n-k} x^{k}, \quad e_{n}(x ; q)=\sum_{k=0}^{n}\left[\begin{array}{l}
n \\
k
\end{array}\right]_{q} \tilde{E}_{n-k} x^{k}
$$

Recall that (see [6]) an entire function $f$ has a $p$-exponential growth of order $k$ and a finite type $(p, k \in \mathbb{R}-\{0\}$ with $p>1)$ if there exists a real number $K>0, \alpha$ such that

$$
|f(x)|<K p^{\frac{k}{2}\left(\frac{\log |x|}{\log p}\right)^{2}}|x|^{\alpha} .
$$

The following results from [2] will be needed in the sequel.

Theorem 2.3 Let $0<q<1$ and $f$ be a function of $q^{-1}$-exponential growth of order less than or equal to 1 . Then

$$
f(z)=\sum_{n=0}^{\infty}\left(D_{q^{-1}}^{2 n} f(1) A_{n}(z)-D_{q^{-1}}^{2 n} f(0) B_{n}(z)\right),
$$

where $A_{n}$ and $B_{n}$ are polynomials of degree $2 n+1$ defined by

$$
\begin{aligned}
& A_{n}(z)=\frac{2^{2 n+1}}{[2 n+1] !} \sum_{j=0}^{2 n+1}\left[\begin{array}{c}
2 n+1 \\
j
\end{array}\right]_{q}(-z ; q)_{j} 2^{-j} \beta_{2 n+1-j}, \\
& B_{n}(z)=\frac{2^{2 n+1}}{[2 n+1] !} B_{2 n+1}(z / 2 ; q) .
\end{aligned}
$$

Furthermore, the polynomials $A_{n}$ are defined recursively by $A_{0}(z)=z$ and, for $n \in \mathbb{N}, A_{n}$ satisfies the second order q-difference equation

$$
D_{q^{-1}}^{2} A_{n}(z)=A_{n-1}(z), \quad A_{n}(0)=A_{n}(1)=0 \quad(n \in \mathbb{N}) .
$$

The polynomials $B_{n}$ are defined recursively by $B_{0}(z)=1-z$ and, for $n \in \mathbb{N}, B_{n}$ satisfies the second order q-difference equation

$$
D_{q^{-1}}^{2} B_{n}(z)=B_{n-1}(z), \quad B_{n}(0)=B_{n}(1)=0 \quad(n \in \mathbb{N}) .
$$

Lemma 2.4 Let $z \in \mathbb{C}$. Then

$$
A_{n}(z):=\varepsilon_{q^{-1}}^{1} B_{n}(z)
$$

where $\varepsilon_{q^{-1}}^{y}$ is a q-translation operator defined by

$$
\varepsilon_{q^{-1}}^{y} x^{n}=x^{n}\left(-y / x ; q^{-1}\right)_{n}=q^{-\frac{n(n-1)}{2}} y^{n}(-x / y ; q)_{n} .
$$




\section{The Green's function of a certain $q$-differential system}

In this section, we consider certain boundary value problems which are related to $q$ Lidstone polynomials, and then we define these polynomials by using Green's function.

Consider the following $q$-differential equation:

$$
D_{q^{-1}}^{2} y(x)-f(x)=0 \quad\left(x \in A_{q}^{*}\right),
$$

subject to the boundary conditions

$$
y(0)=0, \quad y(1)=0 .
$$

Theorem 3.1 The boundary value problem (3.1)-(3.2) is equivalent to the basic Fredholm q-integral equation

$$
y(x)=\int_{0}^{1} G\left(x, q^{2} t\right) f\left(q^{2} t\right) d_{q} t
$$

where

$$
G(x, t)= \begin{cases}-t(1-x), & 0 \leq t<x \leq 1 \\ -x(q-t), & 0 \leq x<t \leq 1\end{cases}
$$

Proof Since $D_{q^{-1}}^{2} y(x)=\frac{1}{q}\left(D_{q}^{2} y\right)\left(\frac{x}{q^{2}}\right)$, Equation (3.1) can be written as

$$
D_{q}^{2} y(x)-q f\left(q^{2} x\right)=0 \quad\left(x \in A_{q}^{*}\right) .
$$

By taking double $q$-integral for (3.5), we obtain

$$
y(x)=q \int_{0}^{x}(x-q t) f\left(q^{2} t\right) d_{q} t+c_{1} x+c_{2},
$$

where $c_{1}$ and $c_{2}$ are arbitrary constants. Now, using the boundary conditions, we get

$$
c_{1}=-q \int_{0}^{1}(1-q t) f\left(q^{2} t\right) d_{q} t \quad \text { and } \quad c_{2}=0 .
$$

Substituting in (3.6), we obtain the required result.

Now, consider the following equations:

$$
\begin{aligned}
G_{1}\left(x, q^{2} t\right) & :=G\left(x, q^{2} t\right) \\
G_{n}\left(x, q^{2} t\right) & =\int_{0}^{1} G\left(x, q^{2} y\right) G_{n-1}\left(q^{2} y, q^{2} t\right) d_{q} y \quad(n=2,3, \ldots) \\
& =\int_{0}^{1} \cdots \int_{0}^{1} G\left(x, q^{2} t_{1}\right) G\left(q^{2} t_{1}, q^{2} t_{2}\right) \cdots G\left(q^{2} t_{n-1}, q^{2} t\right) d_{q} t_{1} d_{q} t_{2} \cdots d_{q} t_{n-1} .
\end{aligned}
$$


Corollary 3.2 The q-Lidstone polynomials $A_{m}$ and $B_{m}$ are given by

$$
\begin{aligned}
& A_{0}(x)=x, \\
& A_{m}(x)=\int_{0}^{1} G\left(x, q^{2} t\right) A_{m-1}\left(q^{2} t\right) d_{q} t=q^{2} \int_{0}^{1} t G_{m}\left(x, q^{2} t\right) d_{q} t,
\end{aligned}
$$

and

$$
\begin{aligned}
& B_{0}(x)=1-x \\
& B_{m}(x)=\int_{0}^{1} G\left(x, q^{2} t\right) B_{m-1}\left(q^{2} t\right) d_{q} t=\int_{0}^{1} G_{m}\left(x, q^{2} t\right)\left(1-q^{2} t\right) d_{q} t .
\end{aligned}
$$

Proof The proof follows immediately from Theorem 3.1, Equation (3.7), Equation (2.4) and Equation (2.5).

Theorem 3.3 Let $0<q<1$ and $g \in C^{2 n}\left(A_{q}^{*}\right)$. Then

$$
g(x)=\sum_{m=0}^{n-1}\left[D_{q^{-1}}^{2 m} g(1) A_{m}(x)-D_{q^{-1}}^{2 m} g(0) B_{m}(x)\right]+\int_{0}^{1} G_{n}\left(x, q^{2} t\right) D_{q^{-1}}^{2 n} g\left(q^{2} t\right) d_{q} t
$$

where $A_{m}$ and $B_{m}$ are $q$-Lidstone polynomials of degree $2 m+1$.

Proof From Theorem 3.1 we can verify that, for $q \in(0,1)$ and $g \in C^{2 n}\left(A_{q}^{*}\right)$, the $q$-integral equation

$$
g(x)=\int_{0}^{1} G_{n}\left(x, q^{2} t\right) f\left(q^{2} t\right) d_{q} t
$$

is the solution of the $q$-differential system

$$
\left\{\begin{array}{l}
D_{q^{-1}}^{2 n} g(x)-f(x)=0 \quad\left(x \in A_{q}^{*}\right) \\
D_{q^{-1}}^{2 k} g(0)=D_{q^{-1}}^{2 k} g(1)=0 \quad(k=0,1, \ldots, n-1)
\end{array}\right.
$$

Furthermore, the unique solution of the system

$$
\left\{\begin{array}{l}
D_{q^{-1}}^{2 n} g(x)-f(x)=0 \quad\left(x \in A_{q}^{*}\right), \\
D_{q^{-1}}^{2 k} g(0)=a_{k}, \quad D_{q^{-1}}^{2 k} g(1)=b_{k} \quad(k=0,1, \ldots, n-1)
\end{array}\right.
$$

is

$$
\begin{aligned}
g(x)= & a_{0}(x-1)+b_{0} x+\sum_{k=1}^{n-1} a_{k} \int_{0}^{1}\left(q^{2} t-1\right) G_{k}\left(x, q^{2} t\right) d_{q} t \\
& +\sum_{k=1}^{n-1} b_{k} \int_{0}^{1} q^{2} t G_{k}\left(x, q^{2} t\right) d_{q} t+\int_{0}^{1} G_{n}\left(x, q^{2} t\right) f\left(q^{2} t\right) d_{q} t .
\end{aligned}
$$


Replacing $a_{k}, b_{k}$ and $f(x)$ by their values in terms of $g(x)$ as given by the $q$-differential system (3.10), we get

$$
\begin{aligned}
g(x)= & g(0)(x-1)+g(1) x+\sum_{k=1}^{n-1} D_{q^{-1}}^{2 k} g(0) \int_{0}^{1}\left(q^{2} t-1\right) G_{k}\left(x, q^{2} t\right) d_{q} t \\
& +\sum_{k=1}^{n-1} D_{q^{-1}}^{2 k} g(1) \int_{0}^{1} q^{2} t G_{k}\left(x, q^{2} t\right) d_{q} t+\int_{0}^{1} G_{n}\left(x, q^{2} t\right) D_{q^{-1}}^{2 n} g\left(q^{2} t\right) d_{q} t .
\end{aligned}
$$

Therefore, according to Equations (3.8) and (3.9), we obtain the required result.

Remark 3.4 By using Theorem 3.3, and from Equations (2.4) and (2.5), we have

$$
\begin{aligned}
D_{q^{-1}}^{2 j} g(x)= & \sum_{m=j}^{n-1}\left[D_{q^{-1}}^{2 m} g(1) D_{q^{-1}}^{2 j} A_{m}(x)+D_{q^{-1}}^{2 m} g(0) D_{q^{-1}}^{2 j} B_{m}(x)\right] \\
& +\int_{0}^{1} G_{n-j}\left(x, q^{2} t\right) D_{q^{-1}}^{2 n} g(t) d_{q} t \\
= & \sum_{m=j}^{n-1}\left[D_{q^{-1}}^{2 m} g(1) A_{m-j}(x)+D_{q^{-1}}^{2 m} g(0) B_{m-j}(x)\right] \\
& +\int_{0}^{1} G_{n-j}\left(x, q^{2} t\right) D_{q^{-1}}^{2 n} g(t) d_{q} t \\
= & \sum_{m=0}^{n-j-1}\left[D_{q^{-1}}^{2(m+j)} g(1) A_{m}(x)+D_{q^{-1}}^{2(m+j)} g(0) B_{m}(x)\right] \\
& +\int_{0}^{1} G_{n-j}\left(x, q^{2} t\right) D_{q^{-1}}^{2 n} g(t) d_{q} t, \\
& +\int_{0}^{n-j-1}\left[D_{q^{-1}, x}^{2(m+j)} g(1) G_{n-j}\left(x, q^{2} t\right) D_{q^{-1}}^{2 n} g(t) d_{q} t .\right.
\end{aligned}
$$

\section{Certain $q$-Fourier expansions}

The purpose of this section is to obtain the $q$-Fourier series expansions of the following $q$-integrals:

$$
\int_{0}^{1}\left(q^{2} t\right)^{k} G_{n}\left(x, q^{2} t\right) d_{q} t, \quad k=0,1, n \leq 4
$$

and then to compute the series expansions of some of $q$-Lidstone polynomials which will be used to solve the boundary value problem (1.1)-(1.2). 
First, recall that the $q$-trigonometric functions $C_{q}(z)$ and $S_{q}(z)$ are defined for $z \in \mathbb{C}$ by

$$
\begin{aligned}
& C_{q}(z)=\sum_{n=0}^{\infty}(-1)^{n} \frac{q^{n(n-1 / 2)} z^{2 n}}{(q ; q)_{2 n}}=\frac{z}{1-q}{ }_{1} \phi_{1}\left(0 ; q ; q^{2}, q^{1 / 2} z^{2}\right), \\
& S_{q}(z)=\sum_{n=0}^{\infty}(-1)^{n} \frac{q^{n(n+1 / 2)} z^{2 n+1}}{(q ; q)_{2 n+1}}=\frac{z}{1-q}{ }_{1} \phi_{1}\left(0 ; q^{3} ; q^{2}, q^{3 / 2} z^{2}\right) .
\end{aligned}
$$

The Fourier series expansion for any function defined on the $q$-linear grid $\mathcal{A}_{q}$ is the following (see $[7,8])$ :

$$
S_{q}(f):=\frac{a_{0}}{2}+\sum_{k=1}^{\infty}\left[a_{k} C_{q}\left(q^{1 / 2} w_{k} z\right)+b_{k} S_{q}\left(q w_{k} z\right)\right]
$$

where $a_{0}=\int_{-1}^{1} f(t) d_{q} t$ and, for $k=1,2, \ldots$,

$$
\begin{aligned}
& a_{k}=\frac{1}{\mu_{k}} \int_{-1}^{1} f(t) C_{q}\left(q^{1 / 2} w_{k} t\right) d_{q} t, \quad b_{k}=\frac{\sqrt{q}}{\mu_{k}} \int_{-1}^{1} f(t) S_{q}\left(q w_{k} t\right) d_{q} t, \\
& \mu_{k}=(1-q) C_{q}\left(q^{1 / 2} w_{k}\right) S_{q}^{\prime}\left(w_{k}\right)
\end{aligned}
$$

on the $q$-linear grid $\mathcal{A}_{q}$, where $\left\{w_{k}: k \in \mathbb{N}\right\}$ is the set of positive zeroes of $S_{q}(z)$.

One can verify that

$$
D_{q, z} C_{q}(w z)=-\frac{w}{1-q} S_{q}(w z \sqrt{q}) \quad \text { and } \quad D_{q, z} S_{q}(w z)=\frac{w}{1-q} C_{q}(w z \sqrt{q}) .
$$

Lemma 4.1 Let $x \in A_{q}^{*}$ and $n \in \mathbb{N}$. Then

$$
\int_{0}^{1} G\left(x, q^{2} y\right) S_{q}\left(q^{n} w_{k} y\right) d_{q} y=\frac{(1-q)^{2}}{q^{2 n-5 / 2} w_{k}^{2}}\left(x S_{q}\left(q^{n-1} w_{k}\right)-S_{q}\left(q^{n-1} w_{k} x\right)\right) .
$$

Proof By using Equations (3.1) and (3.3), the $q$-integral

$$
y(x)=\int_{0}^{1} G_{1}\left(x, q^{2} y\right) S_{q}\left(q^{n} w_{k} y\right) d_{q} y
$$

is the solution of the $q$-differential system

$$
\left\{\begin{array}{l}
D_{q^{-1}}^{2} y(x)-S_{q}\left(q^{n-2} w_{k} x\right)=0 \quad\left(x \in A_{q}^{*}\right) \\
y(0)=0, \quad y(1)=0
\end{array}\right.
$$

Therefore,

$$
\begin{aligned}
& D_{q} y(x)=\frac{-(1-q)}{q^{n-\frac{3}{2}} w_{k}} C_{q}\left(q^{n-\frac{1}{2}} w_{k} x\right)+c_{1}, \\
& y(x)=\frac{-(1-q)^{2}}{q^{2 n-\frac{5}{2}} w_{k}^{2}} S_{q}\left(q^{n-1} w_{k} x\right)+c_{1} x+c_{2} .
\end{aligned}
$$


From the boundary conditions, we get

$$
c_{1}=\frac{(1-q)^{2}}{q^{2 n-\frac{5}{2}} w_{k}^{2}} S_{q}\left(q^{n-1} w_{k}\right) \quad \text { and } \quad c_{2}=0
$$

Substituting the values of $c_{1}$ and $c_{2}$ into Equation (4.2), we obtain the required result.

Lemma 4.2 For $x \in A_{q}^{*}$, the following $q$-Fourier series expansion holds:

$$
\int_{0}^{1} G\left(x, q^{2} t\right) d_{q} t=-2 \sqrt{q}(1-q)^{2} \sum_{k=1}^{\infty} \frac{L_{k}}{w_{k}^{2}} S_{q}\left(w_{k} x\right)
$$

where

$$
L_{k}:=\frac{1-C_{q}\left(q^{1 / 2} w_{k}\right)}{w_{k} C_{q}\left(q^{1 / 2} w_{k}\right) S_{q}^{\prime}\left(w_{k}\right)}
$$

Proof By computing the $q$-Fourier series expansion of the function $f(x)=1$ for $0<x<1$, we get

$$
1=2 \sum_{k=1}^{\infty} \frac{1-C_{q}\left(q^{1 / 2} w_{k}\right)}{w_{k} C_{q}\left(q^{1 / 2} w_{k}\right) S_{q}^{\prime}\left(w_{k}\right)} S_{q}\left(q w_{k} t\right), \quad t \in A_{q}^{*} .
$$

Multiplying (4.4) by $G_{1}\left(x, q^{2} t\right)$ and integrating with respect to $t$ from zero to unity, we get

$$
\int_{0}^{1} G_{1}\left(x, q^{2} t\right) d_{q} t=2 \sum_{k=1}^{\infty} L_{k} \int_{0}^{1} G_{1}\left(x, q^{2} t\right) S_{q}\left(w_{k} q t\right) d_{q} t
$$

where

$$
L_{k}:=\frac{1-C_{q}\left(q^{1 / 2} w_{k}\right)}{w_{k} C_{q}\left(q^{1 / 2} w_{k}\right) S_{q}^{\prime}\left(w_{k}\right)}, \quad x \in A_{q}^{*} .
$$

By using Lemma 4.1, we get

$$
\int_{0}^{1} G_{1}\left(x, q^{2} t\right) S_{q}\left(w_{k} q t\right) d_{q} t=\frac{-\sqrt{q}(1-q)^{2}}{w_{k}^{2}} S_{q}\left(w_{k} x\right) .
$$

Substituting from (4.6) into (4.5), we obtain the required series.

Lemma 4.3 For $x \in A_{q}^{*}$, the following $q$-Fourier series expansion holds:

$$
\int_{0}^{1} G\left(x, q^{2} t\right)\left(q^{2} t\right) d_{q} t=-2 q^{5 / 2}(1-q)^{2} \sum_{k=1}^{\infty} \frac{\widetilde{L_{k}}}{w_{k}^{2}} S_{q}\left(w_{k} x\right) \text {, }
$$

where

$$
\widetilde{L_{k}}:=\frac{q w_{k} C_{q}\left(q^{1 / 2} w_{k}\right)-(1-q) S_{q}\left(q w_{k}\right)}{q^{2} w_{k}^{2} C_{q}\left(q^{1 / 2} w_{k}\right) S_{q}^{\prime}\left(w_{k}\right)}
$$


Proof Considering the function $g(t)=t$ for $0<t<1$ and computing the $q$-Fourier series of the extension of $g$ as an odd function on $[-1,1]$, we get

$$
t=2 \sum_{k=1}^{\infty} \widetilde{L_{k}} S_{q}\left(q w_{k} t\right) \quad \text { for all } 0<t<1
$$

where

$$
\widetilde{L_{k}}:=\frac{q w_{k} C_{q}\left(q^{1 / 2} w_{k}\right)-(1-q) S_{q}\left(q w_{k}\right)}{q^{2} w_{k}^{2} C_{q}\left(q^{1 / 2} w_{k}\right) S_{q}^{\prime}\left(w_{k}\right)} .
$$

Hence, the proof can be performed by using (4.7) similar to the proof of Lemma 4.2. So, we will omit it.

Throughout the following results, we define the constants $L_{k}$ and $\widetilde{L_{k}}$ as in Lemma 4.3 and Lemma 4.3, respectively.

Note that, by using Equation (3.7), we get

$$
G_{2}\left(x, q^{2} t\right)=\int_{0}^{1} G\left(x, q^{2} y\right) G\left(q^{2} y, q^{2} t\right) d_{q} y
$$

Integrating (4.9) with respect to $t$ from 0 to unity and using Lemma 4.2, we obtain

$$
\int_{0}^{1} G_{2}\left(x, q^{2} t\right) d_{q} t=-2 \sqrt{q}(1-q)^{2} \sum_{k=1}^{\infty} \frac{L_{k}}{w_{k}^{2}} \int_{0}^{1} G\left(x, q^{2} y\right) S_{q}\left(q^{2} w_{k} y\right) d_{q} y .
$$

Again, using Lemma 4.1, we get

$$
\int_{0}^{1} G\left(x, q^{2} y\right) S_{q}\left(q^{2} w_{k} y\right) d_{q} y=\frac{(1-q)^{2}}{q^{3 / 2} w_{k}^{2}}\left(x S_{q}\left(q w_{k}\right)-S_{q}\left(q w_{k} x\right)\right) .
$$

Hence,

$$
\int_{0}^{1} G_{2}\left(x, q^{2} t\right) d_{q} t=-2 \frac{(1-q)^{4}}{q} \sum_{k=1}^{\infty} \frac{L_{k}}{w_{k}^{4}}\left(x S_{q}\left(q w_{k}\right)-S_{q}\left(q w_{k} x\right)\right) .
$$

Repeating the process for $n=3$ and $n=4$, we obtain the following result.

Theorem 4.4 For $x \in A_{q}^{*}$ and $n \leq 4$, the following expansion holds:

$$
\begin{aligned}
q^{2} \int_{0}^{1} G_{n}\left(x, q^{2} t\right) d_{q} t= & \frac{(-1)^{n-1}(1-q)^{2 n}}{q^{n(n-3 / 2)}}\left[\sum_{k=1}^{\infty} \frac{L_{k}}{w_{k}^{2 n}}\left(x S_{q}\left(w_{k} q^{n-1}\right)-S_{q}\left(w_{k} q^{n-1} x\right)\right)\right. \\
& +2 \sum_{i=1}^{n-2}(-1)^{i} q^{2(n+i-1)} \sum_{k=1}^{\infty} \frac{L_{k}}{w_{k}^{2(n-i)}} S_{q}\left(q^{n-i-1} w_{k}\right) \\
& \left.\times \sum_{k=1}^{\infty} \frac{\widetilde{L_{k}}}{w_{k}^{2 i}}\left(x S_{q}\left(q^{i-1} w_{k}\right)-S_{q}\left(q^{i-1} w_{k} x\right)\right)\right]
\end{aligned}
$$


Remark 4.5 In the classical case, Widder [9] concluded a general formula for a Fourier series of the integral of Green's functions $G_{n}$ for all $n \in \mathbb{N}$. Theorem 4.4 gives a formula for the $q$-Fourier series of $\int_{0}^{1} G_{n}\left(x, q^{2} t\right) d_{q} t$ for $n \leq 4$, we could not put it in a closed form for all $n \in \mathbb{N}$. However, we can verify that

$$
\int_{0}^{1} G_{n}\left(x, q^{2} t\right) d_{q} t=\frac{(-1)^{n-1}(1-q)^{2 n}}{q^{n(n-3 / 2)}} S_{k, n} \quad(n \in \mathbb{N}),
$$

where $S_{k, n}$ denotes a sum of $q$-series which converge uniformly on $A_{q}^{*}$ and depend on the $q$-trigonometric function $S_{q}$ and the constants $L_{k}$ and $\widetilde{L_{k}}$.

Theorem 4.6 For $x \in A_{q}^{*}$ and $n \leq 4$, the following expansion holds:

$$
\begin{aligned}
\int_{0}^{1} G_{n}\left(x, q^{2} t\right) t d_{q} t= & \frac{(-1)^{n-1}(1-q)^{2 n}}{q^{n(n-3 / 2)}}\left[\sum_{k=1}^{\infty} \frac{\widetilde{L_{k}}}{w_{k}^{2 n}}\left(x S_{q}\left(w_{k} q^{n-1}\right)-S_{q}\left(w_{k} q^{n-1} x\right)\right)\right. \\
& +2 \sum_{i=1}^{n-2}(-1)^{i} q^{2(n+i-1)} \sum_{k=1}^{\infty} \frac{\widetilde{L_{k}}}{w_{k}^{2(n-i)}} S_{q}\left(q^{n-i-1} w_{k}\right) \\
& \left.\times \sum_{k=1}^{\infty} \frac{\widetilde{L_{k}}}{w_{k}^{2 i}}\left(x S_{q}\left(q^{i-1} w_{k}\right)-S_{q}\left(q^{i-1} w_{k} x\right)\right)\right] .
\end{aligned}
$$

Proof The proof is similar to the proof of Theorem 4.4 and is omitted.

The following corollary follows immediately from Theorems 4.4 and 4.6.

Corollary 4.7 For $x \in A_{q}^{*}$ and $n \leq 4$, the following expansion holds:

$$
\begin{aligned}
& \int_{0}^{1} G_{n}\left(x, q^{2} t\right)\left(1-q^{2} t\right) d_{q} t \\
& =\frac{(-1)^{n-1}(1-q)^{2 n}}{q^{n(n-3 / 2)}}\left[\sum_{k=1}^{\infty} \frac{1}{w_{k}^{2 n}}\left(L_{k}-q^{2} \widetilde{L_{k}}\right)\left(x S_{q}\left(w_{k} q^{n-1}\right)-S_{q}\left(w_{k} q^{n-1} x\right)\right)\right. \\
& \quad+2 \sum_{i=1}^{n-2}(-1)^{i} q^{2(n+i-1)} \sum_{k=1}^{\infty} \frac{S_{q}\left(q^{n-i-1} w_{k}\right)}{w_{k}^{2(n-i)}}\left(L_{k}-q^{2} \widetilde{L_{k}}\right) \\
& \left.\quad \times \sum_{k=1}^{\infty} \frac{\widetilde{L_{k}}}{\widetilde{w_{k}^{2 i}}}\left(x S_{q}\left(q^{i-1} w_{k}\right)-S_{q}\left(q^{i-1} w_{k} x\right)\right)\right] .
\end{aligned}
$$

Corollary 4.8 For $x \in A_{q}^{*}$ and $n \leq 4$, the $q$-Fourier series for the q-Lidstone polynomials $A_{n}(x)$ and $B_{n}(x)$ are given by

$$
\begin{aligned}
A_{n}(x)= & \frac{(-1)^{n-1}(1-q)^{2 n}}{q^{n(n-3 / 2)}}\left[\sum_{k=1}^{\infty} \frac{\widetilde{L_{k}}}{w_{k}^{2 n}}\left(x S_{q}\left(w_{k} q^{n-1}\right)-S_{q}\left(w_{k} q^{n-1} x\right)\right)\right. \\
& +2 \sum_{i=1}^{n-2}(-1)^{i} q^{2(n+i-1)} \sum_{k=1}^{\infty} \frac{\widetilde{L_{k}}}{w_{k}^{2(n-i)}} S_{q}\left(q^{n-i-1} w_{k}\right) \\
& \left.\times \sum_{k=1}^{\infty} \frac{\widetilde{L_{k}}}{w_{k}^{2 i}}\left(x S_{q}\left(q^{i-1} w_{k}\right)-S_{q}\left(q^{i-1} w_{k} x\right)\right)\right]
\end{aligned}
$$




$$
\begin{aligned}
B_{n}(x)= & \frac{(-1)^{n-1}(1-q)^{2 n}}{q^{n(n-3 / 2)}}\left[\sum_{k=1}^{\infty} \frac{1}{w_{k}^{2 n}}\left(L_{k}-q^{2} \widetilde{L_{k}}\right)\left(x S_{q}\left(w_{k} q^{n-1}\right)-S_{q}\left(w_{k} q^{n-1} x\right)\right)\right. \\
& +2 \sum_{i=1}^{n-2}(-1)^{i} q^{2(n+i-1)} \sum_{k=1}^{\infty} \frac{S_{q}\left(q^{n-i-1} w_{k}\right)}{w_{k}^{2(n-i)}}\left(L_{k}-q^{2} \widetilde{L_{k}}\right) \\
& \left.\times \sum_{k=1}^{\infty} \frac{\widetilde{L_{k}}}{w_{k}^{2 i}}\left(x S_{q}\left(q^{i-1} w_{k}\right)-S_{q}\left(q^{i-1} w_{k} x\right)\right)\right] .
\end{aligned}
$$

Proof It follows immediately from Theorem 4.6, Corollary 4.7, Equations (3.8) and (3.9).

Proposition 4.9 There exists a constant $C$ such that

$$
0 \leq(-1)^{n} \int_{0}^{1} G_{n}\left(x, q^{2} t\right) d_{q} t \leq \frac{(1-q)^{2 n}}{q^{n(n-3 / 2)}} C .
$$

Proof By using Equations (3.4) and (3.7), we get

$$
(-1)^{n} \int_{0}^{1} G_{n}\left(x, t q^{-1}\right) d_{q} t \geq 0 .
$$

Another inequality follows from Theorem 4.4 together with the result that the series in (4.10) converges uniformly at each fixed point $x \in A_{q}^{*}$.

Proposition 4.10 There exists a constant $\widetilde{C}$ such that

$$
\int_{0}^{1}\left|D_{q^{-1}, x} G_{n}\left(x, q^{2} t\right)\right| d_{q} t \leq \frac{(1-q)^{2(n-1)}}{q^{(n-1)(n-5 / 2)}} \widetilde{C} .
$$

Proof By using (3.7), we have

$$
\begin{aligned}
\int_{0}^{1}\left|D_{q^{-1}, x} G_{n}\left(x, q^{2} t\right)\right| d_{q} t= & \int_{0}^{1}\left[D_{q^{-1, x}} \int_{0}^{1} G\left(x, q^{2} y\right)(-1)^{n-1} G_{n-1}\left(q^{2} y, q^{2} t\right) d_{q} y\right] d_{q} t \\
= & \int_{0}^{1} \int_{0}^{x}(-1)^{n-1}\left(q^{2} y\right) G_{n-1}\left(q^{2} y, q^{2} t\right) d_{q} y d_{q} t \\
& -\int_{0}^{1} \int_{x}^{1}(-1)^{n-1}\left(q-q^{2} y\right) G_{n-1}\left(q^{2} y, q^{2} t\right) d_{q} y d_{q} t .
\end{aligned}
$$

Interchanging the order of the double $q$-integrations and using Proposition 4.9, we get

$$
\begin{aligned}
\int_{0}^{1}\left|D_{q^{-1}, x} G_{n}\left(x, q^{2} t\right)\right| d_{q} t= & \int_{0}^{x}\left(q^{2} y\right)\left[\int_{0}^{1}\left|G_{n-1}\left(q^{2} y, q^{2} t\right)\right| d_{q} t\right] d_{q} y \\
& -\int_{x}^{1}\left(q-q^{2} y\right)\left[\int_{0}^{1}\left|G_{n-1}\left(q^{2} y, q^{2} t\right)\right| d_{q} t\right] d_{q} y \\
\leq & \frac{(1-q)^{2(n-1)}}{q^{(n-1)(n-5 / 2)}} C\left[\int_{0}^{x}\left(q^{2} y\right) d_{q} y-\int_{x}^{1}\left(q-q^{2} y\right) d_{q} y\right] \\
& =\frac{(1-q)^{2(n-1)}}{q^{(n-1)(n-5 / 2)}} C\left[q(1-x)+\frac{q^{2}}{(q+1)}\right] \\
\leq & \frac{(1-q)^{2(n-1)}}{q^{(n-1)(n-5 / 2)}} C\left[q+\frac{q^{2}}{(1+q)}\right] .
\end{aligned}
$$


Hence, if we define the constant $\widetilde{C}$ as

$$
\widetilde{C}:=\left(q+\frac{q^{2}}{(1+q)}\right) C
$$

we get the required result.

We end this section by computing the $q$-Fourier expansion of the $q$-Euler polynomials of degree 2 . We start by the following lemma.

\section{Lemma 4.11}

$$
\sum_{k=1}^{\infty} \frac{L_{k}}{w_{k}}=\frac{\sqrt{q}}{2\left(1-q^{2}\right)} \quad \text { and } \quad \sum_{k=1}^{\infty} \frac{\widetilde{L_{k}}}{w_{k}}=-\frac{\sqrt{q}}{2[3] !(1-q)} .
$$

Proof By computing the $q$-Fourier series for the function $f(x)=|x|$, we obtain

$$
f(x)=\frac{1}{1+q}-\frac{2(1-q)}{\sqrt{q}} \sum_{k=1}^{\infty} \frac{1-C_{q}\left(q^{1 / 2} w_{k}\right)}{w_{k}^{2} C_{q}\left(q^{1 / 2} w_{k}\right) S_{q}^{\prime}\left(w_{k}\right)} C_{q}\left(q^{1 / 2} w_{k} x\right) .
$$

In particular, when $x=0$, this implies

$$
0=\frac{1}{1+q}-\frac{2(1-q)}{\sqrt{q}} \sum_{k=1}^{\infty} \frac{1-C_{q}\left(q^{1 / 2} w_{k}\right)}{w_{k}^{2} C_{q}\left(q^{1 / 2} w_{k}\right) S_{q}^{\prime}\left(w_{k}\right)} .
$$

Therefore,

$$
\sum_{k=1}^{\infty} \frac{L_{k}}{w_{k}}=\frac{\sqrt{q}}{2\left(1-q^{2}\right)}
$$

Similarly, computing the $q$-Fourier series for the function $g(x)=|x|^{2}$, we obtain

$$
|x|^{2}=\frac{1}{[3]}+\frac{2[2](1-q)}{\sqrt{q}} \sum_{k=1}^{\infty} \frac{\widetilde{L_{k}}}{w_{k}} C_{q}\left(q^{1 / 2} w_{k} x\right) .
$$

At $x=0$, we have

$$
\sum_{k=1}^{\infty} \frac{\widetilde{L_{k}}}{w_{k}}=-\frac{\sqrt{q}}{2[3] !(1-q)} .
$$

Theorem 4.12 For $x \in A_{q}^{*}$, the $q$-Fourier series for $q$-Euler polynomials $e_{2}(x ; q)$ is given by

$$
e_{2}(x ; q)=\frac{[2]}{q}\left[-2 \sqrt{q}(1-q)^{2} \sum_{k=1}^{\infty} \frac{L_{k}}{w_{k}^{2}} S_{q}\left(w_{k} x\right)+\left(\frac{q}{1+q}-\frac{q}{2}\right) x\right] .
$$

Proof By using Proposition 2.1, we have

$$
1=e_{0}(x ; q)=D_{q} e_{1}(x ; q) .
$$


Therefore, for $x \in A_{q}^{*}$, the $q$-Fourier expansion of the function $D_{q} e_{1}(x ; q)$ is

$$
D_{q} e_{1}(x ; q)=2 \sum_{k=1}^{\infty} \frac{1-C_{q}\left(q^{1 / 2} w_{k}\right)}{w_{k} C_{q}\left(q^{1 / 2} w_{k}\right) S_{q}^{\prime}\left(w_{k}\right)} S_{q}\left(q w_{k} x\right)
$$

Integrating (4.11) from 0 to $x$, we obtain

$$
e_{1}(x ; q)=\frac{-2(1-q)}{\sqrt{q}} \sum_{k=1}^{\infty} \frac{L_{k}}{w_{k}} C_{q}\left(q^{1 / 2} w_{k} x\right)+C_{1},
$$

where $C$ is a constant of integration. This constant is obtained by putting $x=0$ in Equation (4.12) and then using Lemma 4.11 and the result $e_{1}(0 ; q)=\widetilde{E}_{1}(0)=-\frac{1}{2}$. We get $C_{1}=-\frac{1}{2}+\frac{1}{1+q}$. Hence,

$$
e_{1}(x ; q)=\frac{-2(1-q)}{\sqrt{q}} \sum_{k=1}^{\infty} \frac{L_{k}}{w_{k}} C_{q}\left(q^{1 / 2} w_{k} x\right)+\frac{1}{1+q}-\frac{1}{2} .
$$

Again, using Proposition 2.1 with $n=2$, we get

$$
e_{2}(x ; q)=[2] \int e_{1}(x, q) d_{q} x+C_{2}
$$

Substituting Equation (4.13) into Equation (4.14) gives us

$$
e_{2}(x ; q)=[2]\left[\frac{-2(1-q)}{\sqrt{q}} \sum_{k=1}^{\infty} \frac{L_{k}}{w_{k}} \int C_{q}\left(q^{1 / 2} w_{k} x\right) d_{q} x+\int\left(\frac{1}{1+q}-\frac{1}{2}\right) d_{q} x\right]+C_{2}
$$

This implies

$$
e_{2}(x ; q)=[2]\left[\frac{-2(1-q)^{2}}{\sqrt{q}} \sum_{k=1}^{\infty} \frac{L_{k}}{w_{k}^{2}} S_{q}\left(w_{k} x\right)+\left(\frac{q}{1+q}-\frac{q}{2}\right) x\right]+C_{2} \text {. }
$$

In the last equation putting $x=0$, we get $C_{2}=0$, and hence the theorem.

Corollary 4.13 For $x \in A_{q}^{*}$, the following holds:

$$
e_{2}(x ; q)=\frac{[2]}{q}\left[\int_{0}^{1} G\left(x, q^{2} t\right) d_{q} t+\left(\frac{q}{1+q}-\frac{q}{2}\right) x\right] .
$$

Proof The proof follows immediately from Lemma 4.2 and Theorem 4.12.

Remark 4.14 From Equation (3.9), we have

$$
B_{n}(x)=\int_{0}^{1} G_{n}\left(x, q^{2} t\right) d_{q} t-q^{2} \int_{0}^{1} t G_{n}\left(x, q^{2} t\right) d_{q} t
$$

Thus, by using Corollary 4.13 and Equation (3.8), we obtain the following relation:

$$
B_{1}(x)+q^{2} A_{1}(x)=q\left[\frac{e_{2}(x ; q)}{[2]}+\left(\frac{1}{2}-\frac{1}{1+q}\right) x\right] \text {. }
$$


If $q \rightarrow 1$, Equation (4.15) coincides with the result which is given by Agarwal and Wong [3] in the classical case.

\section{An application: $q$-boundary value problems}

The $q$-difference equations are important in $q$-calculus. This subject initiated in the first quarter of the twentieth century [10-13], and it has been developed over the years. Recently, many authors have studied the existence and uniqueness of solutions for some problems of $q$-difference equations, for instance, see [7, 14-20].

The goal of this section is to solve the boundary value problem (1.1)-(1.2) by using the $q$ Lidstone expansion theorem. The results here attained are the $q$-analogue of those given by Agarwal and Wong [3], where they studied the existence of solutions for

$$
\left\{\begin{array}{l}
(-1)^{n} x^{(2 m)}(t)=f\left(t, x(t), x^{\prime}(t), \ldots, x^{(k)}(t)\right) \\
x^{(2 i)}(0)=a_{i} \\
x^{(2 i)}(1)=b_{i}
\end{array}\right.
$$

where $0 \leq k \leq 2 m-1$ and $i=0,1, \ldots, m-1$ with some conditions imposed on $f$ and $x$.

For our purpose, let us define two constants $C$ and $\widetilde{C}$ as in Proposition 4.9 and Proposition 4.10, respectively, and we introduce the following assumptions:

$H_{1}: K_{j}, 0 \leq j \leq k$ are given real numbers, and define the nonzero constant $M$ to be the maximum of $\left|\phi\left(x, y_{0}, y_{1}, y_{2}, \ldots, y_{k}\right)\right|$ on the compact set $A_{q}^{*} \times E$, where

$$
\begin{aligned}
& E=\left\{\left(y_{0}, y_{1}, y_{2}, \ldots, y_{k}\right),\left|y_{j}\right| \leq 2 K_{j}, 0 \leq j \leq k\right\} . \\
& H_{2}: \frac{(1-q)^{2(n-j)}}{q^{(n-j)(n-j-3 / 2)}} M C \leq K_{2 j}, \quad j=0,1,2, \ldots, \frac{k}{2} ; \\
& H_{3}: \frac{(1-q)^{2(n-j-1)}}{q^{(n-j-1)(n-j-5 / 2)}} M \widetilde{C} \leq K_{2 j+1}, \quad j=0,1,2, \ldots, \frac{k-1}{2} ; \\
& H_{4}: \max \left\{\left|\gamma_{j}\right|,\left|\beta_{j}\right|\right\}+\sum_{i=1}^{n-j-1} \max \left\{\left|\gamma_{i+j}\right|,\left|\beta_{i+j}\right|\right\} \frac{(1-q)^{2 i}}{q^{i(i-3 / 2)}} C \leq K_{2 j} ; \\
& H_{5}:\left|\gamma_{j}+\beta_{j}\right|+\widetilde{C} \sum_{i=1}^{n-j-1} \max \left\{\left|\gamma_{i+j}\right|,\left|\beta_{i+j}\right|\right\} \frac{(1-q)^{2(i-1)}}{q^{(i-1)(i-5 / 2)}} \leq K_{2 j+1} .
\end{aligned}
$$

The proof of the existence results for boundary value problem (1.1)-(1.2) depends on $q$ Lidstone polynomials and the Arzela-Ascoli theorem [21].

Theorem 5.1 Let $q \in(0,1)$ and $y \in C_{q^{-1}}^{n}\left(A_{q}^{*}\right)$ be a real or complex-valued function. Assume that assumptions $H_{1}, H_{2}, H_{3}$ and $H_{4}$ hold. Then the boundary value problem (1.1)-(1.2) has a solution in $E$.

Proof By using Theorem 3.3, we conclude that the boundary value problem (1.1)-(1.2) is equivalent to the following Fredholm $q$-integral equation:

$$
y(x)=\sum_{i=0}^{n-1}\left[\gamma_{i} A_{i}(x)+\beta_{i} B_{i}(x)\right]+\int_{0}^{1} G_{n}\left(x, q^{2} t\right) \phi\left(t, y(t), \ldots, D_{q^{-1}}^{k} y(t)\right) d_{q} t .
$$


Hence, this problem can be interpreted as a fixed point for the mapping $T: C_{q^{-1}}^{k}\left(A_{q}^{*}\right) \rightarrow$ $C_{q^{-1}}^{2 n}\left(A_{q}^{*}\right)$ which is defined by

$$
(T y)(x)=\sum_{i=0}^{n-1}\left[\gamma_{i} A_{i}(x)+\beta_{i} B_{i}(x)\right]+\int_{0}^{1}\left|G_{n}\left(x, q^{2} t\right)\right| \phi\left(t, y(t), \ldots, D_{q^{-1}}^{k} y(t)\right) d_{q} t .
$$

We define the set

$$
J\left(A_{q}^{*}\right):=\left\{y(x) \in C_{q^{-1}}^{k}\left(A_{q}^{*}\right):\left\|D_{q^{-1}}^{j} y\right\|=\max _{0 \leq x \leq 1}\left|D_{q^{-1}}^{j} y(x)\right| \leq 2 K_{j}, 0 \leq j \leq k\right\} .
$$

Notice that $J\left(A_{q}^{*}\right)$ is a closed subset of the space $C_{q^{-1}}^{k}\left(A_{q}^{*}\right)$. We prove that $T$ maps $J\left(A_{q}^{*}\right)$ into itself.

Let $y(x) \in J\left(A_{q}^{*}\right)$. Then, from Equation (5.2), Remark 3.4, Proposition 4.9 and hypotheses $H_{1}, H_{2}$ and $H_{4}$, we get

$$
\begin{aligned}
\left|D_{q^{-1}}^{(2 j)}(T y)(x)\right| \leq & \sum_{i=0}^{n-j-1}\left|\gamma_{i+j} A_{i}(x)+\beta_{i+j} B_{i}(x)\right|+M \int_{0}^{1}\left|G_{n-j}\left(x, q^{2} t\right)\right| d_{q} t \\
\leq & \left|\gamma_{j} x\right|+\left|\beta_{j}(1-x)\right|+\sum_{i=1}^{n-j-1} \mid \gamma_{i+j} \int_{0}^{1}\left(q^{2} t\right) G_{i}\left(x, q^{2} t\right) d_{q} t+\beta_{i+j} \\
& \times \int_{0}^{1}\left(1-q^{2} t\right) G_{i}\left(x, q^{2} t\right) d_{q} t\left|+M \int_{0}^{1}\right| G_{n-j}\left(x, q^{2} t\right) \mid d_{q} t \\
\leq & \sup _{x \in A_{q}^{*}}\left[\left|\gamma_{j} x\right|+\left|\beta_{j}(1-x)\right|\right]+\sum_{i=1}^{n-j-1} \max \left\{\left|\gamma_{i+j}\right|,\left|\beta_{i+j}\right|\right\} \\
& \times \int_{0}^{1}\left|G_{i}\left(x, q^{2} t\right)\right| d_{q} t+M \int_{0}^{1}\left|G_{n-j}\left(x, q^{2} t\right)\right| d_{q} t \\
\leq & \max \left\{\left|\gamma_{j}\right|,\left|\beta_{j}\right|\right\}+\sum_{i=1}^{n-j-1} \max \left\{\left|\gamma_{i+j}\right|,\left|\beta_{i+j}\right|\right\} \frac{(1-q)^{2 i}}{q^{i(i-3 / 2)}} C \\
& +\frac{(1-q)^{2(n-j)}}{q^{(n-j)(n-j-3 / 2)}} M C \leq 2 K_{2 j}, \quad j=0,1,2, \ldots, \frac{k}{2} .
\end{aligned}
$$

Similarly, from Equation (5.2), Remark 3.4, Proposition 4.10 and hypotheses $H_{3}$ and $H_{5}$, we get

$$
\begin{aligned}
\left|D_{q^{-1}}^{(2 j+1)}(T y)(x)\right| \leq & \left|\gamma_{j}+\beta_{j}\right|+\widetilde{C} \sum_{i=1}^{n-j-1} \max \left\{\left|\gamma_{i+j}\right|,\left|\beta_{i+j}\right|\right\} \frac{(1-q)^{2(i-1)}}{q^{(i-1)(i-5 / 2)}} \\
& +\frac{(1-q)^{2(n-j-1)}}{q^{(n-j-1)(n-j-5 / 2)}} M \widetilde{C} \\
\leq & K_{2 j+1}+K_{2 j+1}=2 K_{2 j+1}, \quad j=0,1,2, \ldots, \frac{k-1}{2}
\end{aligned}
$$

This completes the proof of $T\left(J\left(A_{q}^{*}\right)\right) \subseteq J\left(A_{q}^{*}\right)$. Furthermore, from the inequalities (5.3) and (5.4) we conclude that the set

$$
\left\{D_{q^{-1}}^{j}(T) y(x): y(x) \in J\left(A_{q}^{*}\right), 0 \leq j \leq k\right\}
$$


is uniformly bounded and equicontinuous on $J\left(A_{q}^{*}\right)$. Therefore, from the Arzela-Ascoli theorem $\overline{T\left(J\left(A_{q}^{*}\right)\right)}$ is compact. It means that we can find a fixed point of $T$ in $E$ which satisfies the boundary value problem (1.1)-(1.2).

Corollary 5.2 Assume that the function $\phi\left(x, y_{0}, y_{1}, \ldots, y_{k}\right)$ satisfies the following condition on $A_{q}^{*} \times \mathbb{R}^{k+1}$ :

$$
\left|\phi\left(x, y_{0}, y_{1}, \ldots, y_{k}\right)\right| \leq L+\sum_{j=0}^{k} L_{j}\left|y_{j}\right|^{\alpha_{j}}
$$

where $L, L_{j}$ are nonnegative constants, and $0 \leq \alpha_{j}<1$. Then the boundary value problem (1.1)-(1.2) has a solution.

Proof By using (5.5), for $y(x) \in J\left(A_{q}^{*}\right)$, we get

$$
\left|\phi\left(x, y(x), D_{q^{-1}} y(x), D_{q^{-1}}^{2} y(x), \ldots, D_{q^{-1}}^{k} y(x)\right)\right| \leq N
$$

where $N:=L+\sum_{j=0}^{k} L_{j}\left(2 K_{j}\right)^{\alpha_{j}}$. Hence, the result follows by observing that the hypotheses of Theorem 5.1 are satisfied and replacing $M$ by $N$ such that $K_{j}(0 \leq j \leq k)$ are sufficiently large.

\section{Conclusion}

The goal of this paper is to study some properties of $q$-Lidstone polynomials by using Green's function of certain $q$-differential systems and then to solve the following boundary value problem:

$$
\begin{aligned}
& (-1)^{n} D_{q^{-1}}^{2 n} y(x)=\phi\left(x, y(x), D_{q^{-1}} y(x), D_{q^{-1}}^{2} y(x), \ldots, D_{q^{-1}}^{k} y(x)\right), \\
& D_{q^{-1}}^{2 j} y(0)=\beta_{j}, \quad D_{q^{-1}}^{2 j} y(1)=\gamma_{j} \quad\left(\beta_{j}, \gamma_{j} \in \mathbb{C}, j=0,1, \ldots, n-1\right),
\end{aligned}
$$

where $n \in \mathbb{N}$ and $0 \leq k \leq 2 n-1$.

Funding

This research is supported by King Saud University, Saudi Arabia.

Abbreviations

Not applicable.

Availability of data and materials

Not applicable.

Ethics approval and consent to participate

Not applicable.

Competing interests

The authors declare that they have no competing interests.

Consent for publication

Not applicable.

Authors' contributions

The authors read and approved the final manuscript. 


\section{Author details}

${ }^{1}$ Department of Mathematics, Faculty of Science, King Saud University, Riyadh, Kingdom of Saudi Arabia. ${ }^{2}$ Department of Mathematics, Faculty of Science, Cairo University, Cairo, Egypt. ${ }^{3}$ Department of Natural and Engineering Science, Faculty of Applied Studies and Community Service, King Saud University, Riyadh, Kingdom of Saudi Arabia.

\section{Publisher's Note}

Springer Nature remains neutral with regard to jurisdictional claims in published maps and institutional affiliations.

Received: 19 August 2017 Accepted: 14 November 2017 Published online: 22 November 2017

\section{References}

1. Boas, RP, Buck, RC: Polynomial Expansions of Analytic Functions, 2nd edn. Springer, Berlin (1964)

2. Ismail, ME, Mansour, ZS: q-analogues of Lidstone expansion and Bernoulli polynomials (2017, submitted)

3. Agarwal, RP, Wong, PJY: Lidstone polynomials and boundary value problems. Comput. Math. Appl. 17, 1397-1421 (1989)

4. Annaby, MH, Mansour, ZS: q-Fractional Calculus and Equations. Lecture Notes in Mathematics, vol. 2056. Springer, Berlin (2012)

5. Gasper, G, Rahman, M: Basic Hypergeometric Series. Cambridge University Press, Cambrdge (2004)

6. Ramis, JP: About the growth of entire functions solutions of linear algebraic q-difference equations. Ann. Fac. Sci. Toulouse 1(6), 53-94 (1992)

7. Ahmad, B, Nieto, JJ: Basic theory of nonlinear third-order q-difference equations and inclusions. Math. Model. Anal. 18(1), 122-135 (2013)

8. Cardoso, JL: Basic Fourier series: convergence on and outside the $q$-linear grid. J. Fourier Anal. Appl. 17(1), 96-114 (2011)

9. Widder, DV: Completely convex functions and Lidstone series. Trans. Am. Math. Soc. 51, 387-398 (1942)

10. Adams, CR: On the linear ordinary q-difference equation. Ann. Math. 30, 195-205 (1928)

11. Carmichael, RD: The general theory of linear q-difference equations. Am. J. Math. 34, 147-168 (1912)

12. Jackson, FH: On q-difference equations. Am. J. Math. 32, 305-314 (1910)

13. Mason, TE: On properties of the solutions of linear q-difference equations with entire function coefficients. Am. J. Math. 37, 439-444 (1915)

14. Ahmad, B, Alsaedi, A, Ntouyas, SK: A study of second-order q-difference equations with boundary conditions. Adv. Differ. Equ. 2012, Article ID 35 (2012). doi:10.1186/1687-1847-2012-35

15. Annaby, MH, Mansour, ZS: q-Taylor and interpolation series for Jackson q-difference operators. J. Math. Anal. Appl. 344, 472-483 (2008)

16. Bangerezako, G: Variational q-calculus. J. Math. Anal. Appl. 289, 650-665 (2004)

17. Dobrogowska, A, Odzijewicz, A: Second order q-difference equations solvable by factorization method. J. Comput. Appl. Math. 193, 319-346 (2006)

18. El-Shahed, M, Hassan, HA: Positive solutions of q-difference equation. Proc. Am. Math. Soc. 138, 1733-1738 (2010)

19. Ferreira, R: Nontrivial solutions for fractional q-difference boundary value problems. Electron. J. Qual. Theory Differ. Equ. 2010, Article ID 70 (2010)

20. $\mathrm{Ma}, \mathrm{J}$, Yang, J: Existence of solutions for multi-point boundary value problem of fractional $q$-difference equation. Electron. J. Qual. Theory Differ. Equ. 2011, Article ID 92 (2011)

21. Rudin, W: Principles of Mathematical Analysis, 3rd edn. McGraw-Hill, New York (1964)

\section{Submit your manuscript to a SpringerOpen ${ }^{\circ}$ journal and benefit from:}

- Convenient online submission

- Rigorous peer review

Open access: articles freely available online

- High visibility within the field

- Retaining the copyright to your article

Submit your next manuscript at $>$ springeropen.com 